\title{
The first construction phase of the Baikal-GVD neutrino telescope
}

\begin{abstract}
A.D. Avrorin ${ }^{a}$, A.V. Avrorin ${ }^{a}$, V.M. Aynutdinov ${ }^{a}$, R. Bannash ${ }^{g}$, I.A. Belolaptikov ${ }^{b}$, D.Yu. Bogorodskyc, V.B. Brudanin ${ }^{b}$, N.M. Budnev ${ }^{\text {, }}$ I.A. Danilchenko ${ }^{a}$, G.V.Domogatsky ${ }^{a}$, A.A. Doroshenko ${ }^{a}$, A.N. Dyachok ${ }^{c}$, Zh.-A.M. Dzhilkibaev', S.V. Fialkovsky $^{5}$, A.R. Gafarov', O.N. Gaponenko ${ }^{a}$, K.V. Golubkov ${ }^{a}$, T.I. Gress ${ }^{c}$, Z. Honz $^{\text {b }}$, K.G. Kebkal ${ }^{g}$, O.G. Kebkal ${ }^{g}$, K.V. Konischev', A.V. Korobchenko ${ }^{b}$, A.P. Koshechkin $^{\text {a }}$, F.K. Koshel ${ }^{\text {a }}$, A.V. Kozhin ${ }^{d}$, V.F. Kulepov ${ }^{\mathrm{e}}$, D.A. Kuleshov ${ }^{\text {, }}$, V.I. Ljashuk $^{a}$, M.B. Milenin ${ }^{e}$, R.A. Mirgazov', E.R. Osipova ${ }^{d}$, A.I. Panfilov ${ }^{a}$, L.V. Pan'kov', E.N. Pliskovsky $^{b}$, M.I. Rozanov', E.V. Rjabov ${ }^{c}$, B.A. Shaybonov ${ }^{1 b}$, A.A. Sheifler $^{a}$, M.D. Shelepov ${ }^{a}$, A.V. Skurihin ${ }^{d}$, A.A. Smagina ${ }^{b}$, O.V. Suvorova ${ }^{a}$, V.A. Tabolenko $^{c}$, B.A. Tarashanskyc , S.A. Yakovlev ${ }^{\text {, A.V. Zagorodnikov }}{ }^{c}$, V.A. Zhukov ${ }^{\mathrm{a}}$, and V.L. Zurbanov ${ }^{c}$
\end{abstract}

\footnotetext{
${ }^{1}$ Institute for Nuclear Research, 60th October Anniversary pr. 7A, Moscow 117312, Russia

2 Joint Institute for Nuclear Research, Dubna 141980, Russia

${ }^{3}$ Irkutsk State University, Irkutsk 664003, Russia

${ }^{4}$ Skobeltsyn Institute of Nuclear Physics MSU, Moscow 119991, Russia

${ }^{5}$ Nizhni Novgorod State Technical University, Nizhni Novgorod 603950, Russia

${ }^{6}$ St. Petersburg State Marine University, St. Petersburg 190008, Russia

${ }^{7}$ Evologics GmbH, Berlin, Germany
}

Baikal-GVD is a cubic-kilometer scale neutrino telescope, which is currently under construction in Lake Baikal. Array will be formed by multimegaton subarrays - clusters of strings. The first GVD-cluster has been deployed and started operation in April 2015. We describe the design and discuss a capability of GVD-cluster to search for diffuse flux of astrophysical neutrinos recently discovered by IceCube. 


\section{Overview}

The next generation neutrino telescope Baikal-GVD is a kilometer-scale deep underwater Cherenkov detector currently under construction in Lake Baikal [1]. Baikal-GVD is designed to detect astrophysical neutrino fluxes at energies from a few $\mathrm{TeV}$ up to the highest energies of $10^{6}$ $\mathrm{TeV}$ and particularly mapping the high-energy neutrino sky in the Southern Hemisphere including the region of the galactic center.

The site chosen for the experiment is in the southern basin of Lake Baikal. Here, the combination of hydrological, hydro-physical, and landscape factors is optimal for deployment and operation of the neutrino telescope. The water depth is about $1360 \mathrm{~m}$ at distances beginning from about of three kilometers from the shore. The water transparency is characterized by an absorption length of about $20-25 \mathrm{~m}$ and a scattering length of $30-50 \mathrm{~m}$ [2]. The water luminescence is moderate at the detector site.

Baikal-GVD is a 3-dimensional lattice of optical modules (OMs) - photomultiplier tubes housed in transparent pressure spheres. The OMs are arranged at vertical load-carrying cables to form strings. The telescope has a modular structure and consists of clusters of strings functionally independent sub-arrays, which are connected to shore by individual electro-optical cables (see Fig 1).
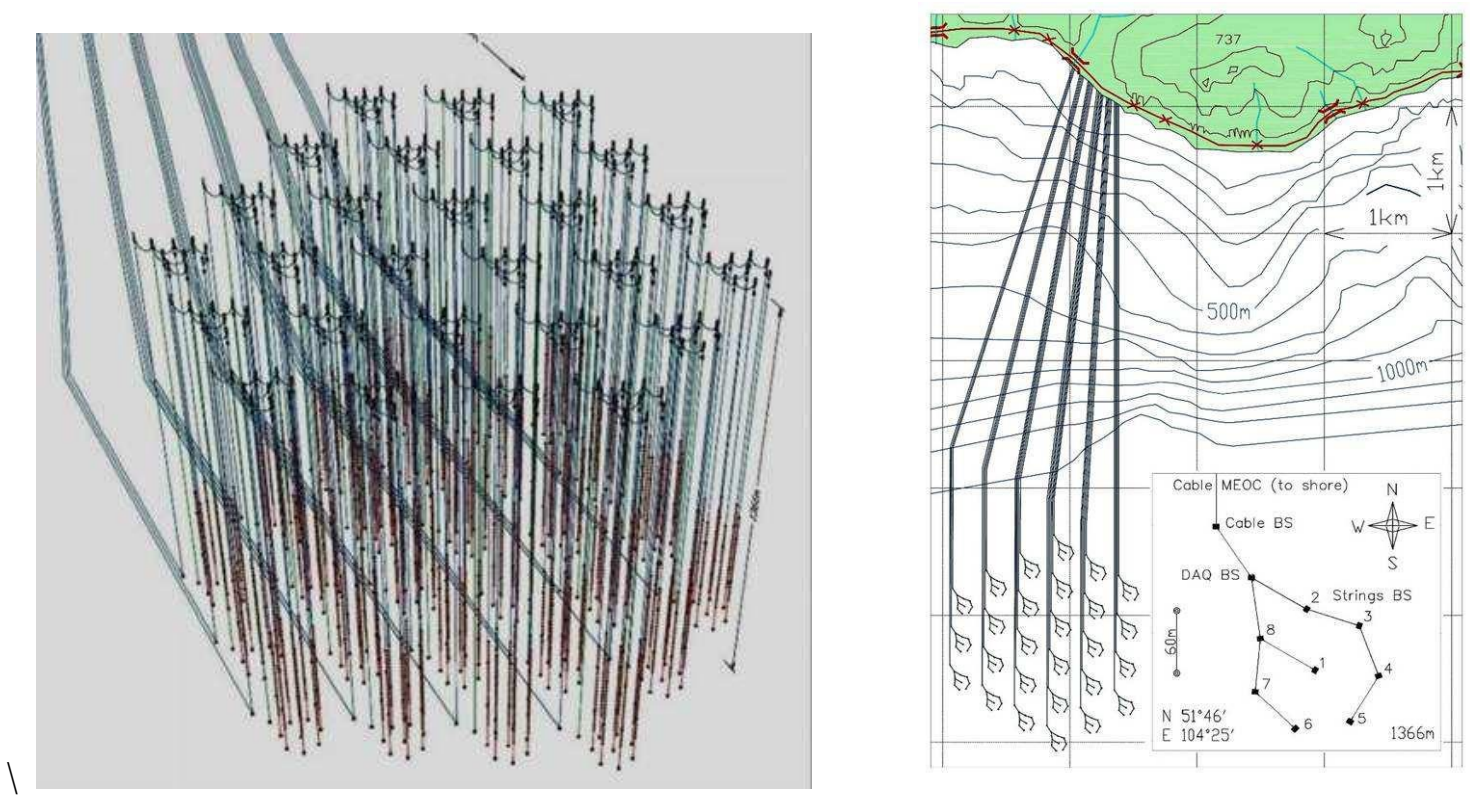

Fig. 1. Left: Artistic view of the GVD-telescope. Right: Layout of the GVD. In inner box layout of the GVD-cluster is shown.

The first prototype of the GVD electronics was deployed in Lake Baikal in April 2008. It was a reduced-size section with six optical modules. This detection unit provided the possibility to study basic elements of the future detector: new optical modules and Flash Analog-to-Digital Converter (FADC) based measuring system [3]. During the next 2 years different versions of prototype string were tested in Lake Baikal as a part of NT200+ detector. The operation of these prototype strings in 2009 and 2010 allows a first assessment of the DAQ performance [4,5]. 

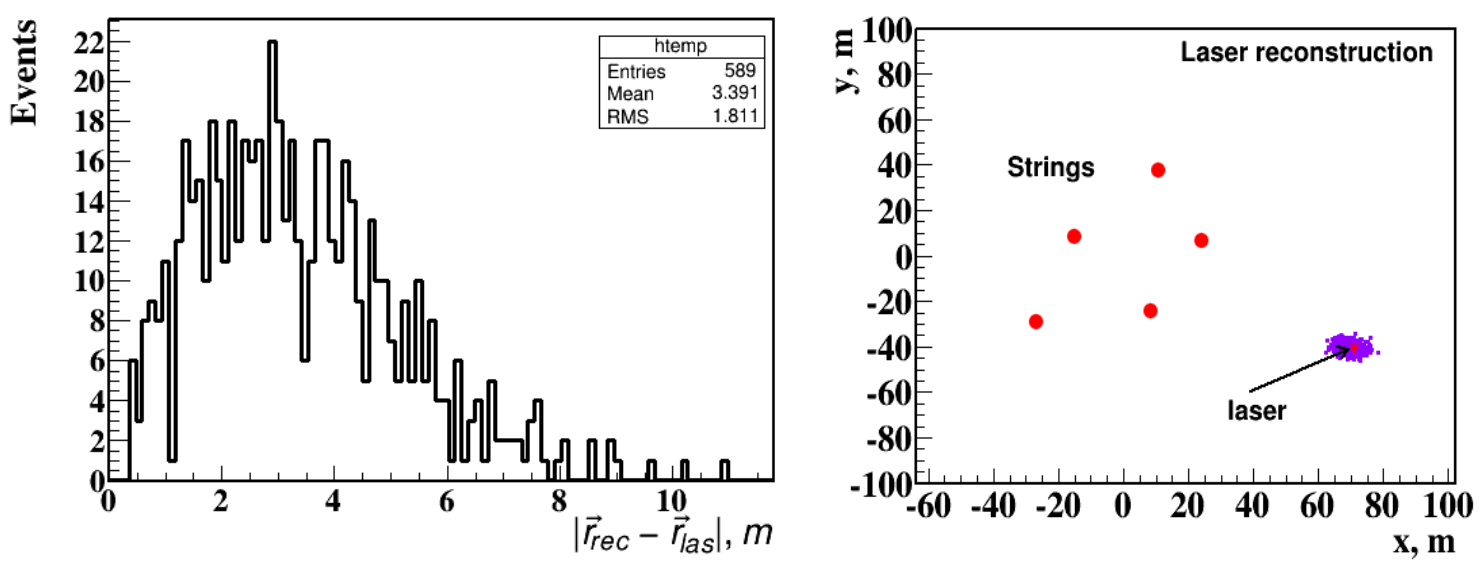

Fig. 2. Left: Deviation of reconstructed laser coordinates from those obtained by the acoustic positioning system. Right: Top view of reconstructed laser positions. The star indicates the laser position obtained by the acoustic system.

The prototyping/early construction phase of the Baikal-GVD project, which aims at construction and operation of the first GVD cluster, has been started in 2011 by deployment and operation of autonomous engineering arrays [6]. The next important step in the realization of the GVD project was made in 2013 by deployment of an enlarged engineering array - the first stage of the first GVD-cluster, which comprises 72 OMs arranged on three $345 \mathrm{~m}$ long strings and operated from April 2013 to February 2014 [1,7]. In 2014 the second stage of the demonstration cluster, which comprises 112 OMs arranged on five strings, as well as an instrumentation string with array calibration and environment monitoring equipment was deployed and operated during 2014 in data taking and testing modes. One of the main goals of the operation in testing mode was to study the quality of in-situ calibration procedures.

The performance and quality of these procedures have been verified by reconstructing position and intensity of a distant calibration laser source. This laser fires five series of $480 \mathrm{~nm}$ light pulses at five fixed intensity levels ranging from approximately $10^{12}$ to $6 \cdot 10^{13}$ photons/pulse. This corresponds to shower energies from about $10 \mathrm{PeV}$ to $600 \mathrm{PeV}$ and allows testing the quality of high-energy cascade reconstruction. The position of the laser source was reconstructed using the arrival times of photons detected by the PMTs, taking into account the timing calibration of the PMTs by LEDs. Results of the reconstruction were compared with the laser coordinates obtained by the acoustic positioning system [8]. Differences between reconstructed coordinates and those obtained by the acoustic system are shown in Fig. 2 (left). The reconstruction accuracy (median value) is about $3 \mathrm{~m}$. Shown in Fig. 2 (right) are strings and laser positions obtained by the acoustic system, as well as reconstructed laser positions. The obtained results prove the expected quality of the array calibration procedures.

\section{The first GVD-cluster}

In April 2015 the first cluster of Baikal-GVD was deployed and started operation in Lake Baikal. This array was named "Dubna". It encloses several Megatons of the clear waters of Lake Baikal. The first cluster of Baikal-GVD comprises a total of 192 optical modules arranged at eight $345 \mathrm{~m}$ long strings, as well as an acoustic positioning system and an instrumentation string with equipment for array calibration and monitoring of environmental parameters. Artistic view of cluster is shown in Fig. 3. Each string comprises 24 OMs spaced by $15 \mathrm{~m}$ at depths of $900 \mathrm{~m}$ to $1250 \mathrm{~m}$ below the surface. In 2015 seven side strings have been located at a reduced radius of $40 \mathrm{~m}$ around a central one (compared to $60 \mathrm{~m}$ for the baseline configuration). The reason is to 
increase the sensitivity to low-energy atmospheric muons and neutrinos which are used for array calibration. In the next year, strings will be moved to the baseline distances.

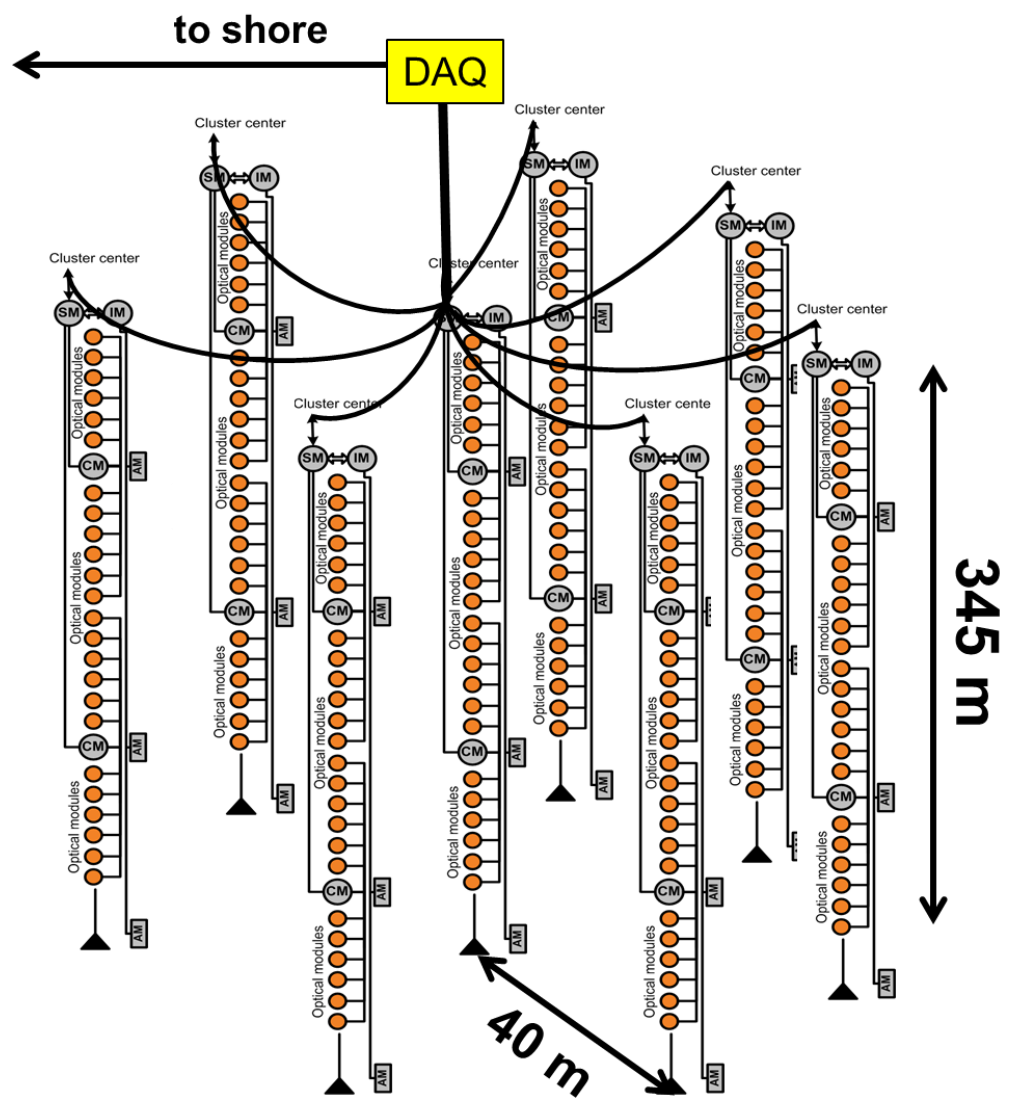

Fig. 3. Artistic view of the first GVD Cluster.

Each optical module consists of a pressure-resistant glass sphere of $43.2 \mathrm{~cm}$ diameter which holds the OM electronics and the PMT which is surrounded by a high permittivity alloy cage for shielding it against the Earth magnetic field. A large photomultiplier tube Hamamatsu R7081-100 with a 10-inch hemispherical photocathode and quantum efficiency up to $35 \%$ has been selected as light sensor. Besides the PMT, an OM comprises a high voltage power supply unit (HV), a fast two-channel preamplifier, and a controller. For time and amplitude calibration of the measuring channel, two LEDs are installed in the optical module. The OM controller is intended for HV control and monitoring for PMT noise measurements and for time and amplitude calibration [9].

The OMs along a string are combined in sections - the functional detection unit of the telescope [10]. Each section includes $12 \mathrm{OMs}$ and the central module (CeM). PMTs signals from all OMs are transmitted through $90 \mathrm{~m}$ long coaxial cables to the CeM of the section, where they are digitized by custom-made ADC boards with $200 \mathrm{MHz}$ sampling rate. The waveform information from all measuring channels of the section is transferred to the Master board located in the CeM. The Master board provides readout of the ADC data, connection via local Ethernet to the cluster DAQ-center, control of the section operation and the section trigger logic. A request analyzer forms the section trigger request (local trigger) on the basis of channel requests 
L (low channel threshold, $\sim 0.3$ p.e.) and $\mathrm{H}$ (high threshold, $\sim 3$ p.e.) from $12 \mathrm{ADC}$ channels. This unit contains a programmable coincidence matrix $(12 \mathrm{Hx} 12 \mathrm{~L})$, which provides a simple way to generate the section trigger request. The basic trigger modes are: (A) coincidences of $>\mathrm{N} \mathrm{L}$ requests within a selectable time window ( $\mathrm{L}>\mathrm{N}$-trigger), or (B) coincidences of $\mathrm{L}$ and $\mathrm{H}$ requests from any neighbouring OMs within a section (L\&H-trigger). A request of the section trigger is transferred from the Master board through a string communication module (CoM) to the cluster DAQ-center, where a global trigger for all sections is generated. Data from the strings are transferred through DSL-modem Ethernet channel to the cluster center. The data transmission between the cluster DAQ-center and shore station is provided through optical fiber lines extended at about $6 \mathrm{~km}$.

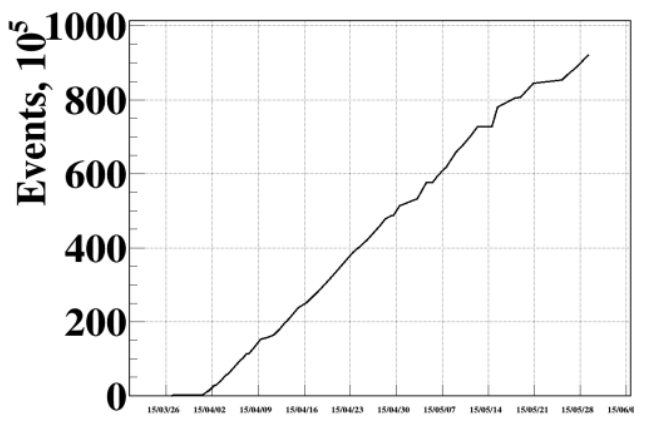

$\mathbf{y y} / \mathbf{m m} / \mathbf{d d}$

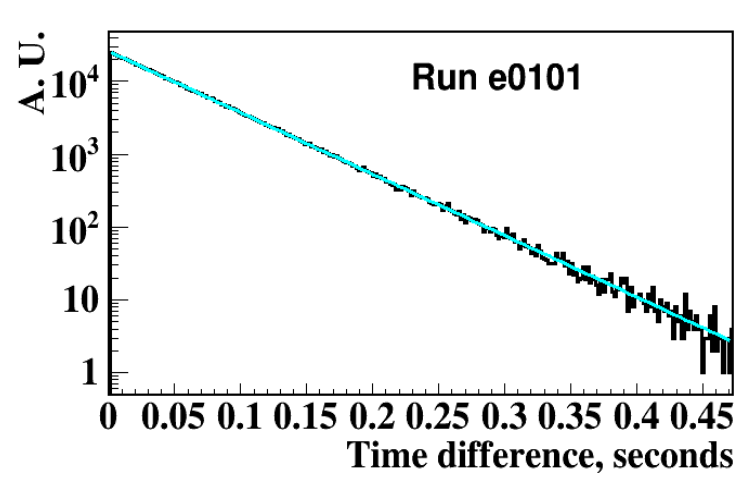

Fig. 4: Left: Integrated number of recorded events since April 2015. Right: The time difference between subsequent events is shown for one run.

The cluster is operating from April 2015 in several testing and data taking modes. Total of $1.7 \times 10^{8}$ events have been recorded from April till June 2015. Figure 4 (left) gives an indication of the data taking efficiency during 2015. Shown in figure 4 (right) is a time difference between subsequent events for one run. An exponential behavior of this distribution is consistent with expectation for randomly distributed experimental events and illustrates quality of data.

The first cluster of Baikal-GVD in its baseline configuration will have the potential to detect astrophysical neutrinos with a flux value measured recently by IceCube $[11,12]$. The search for high-energy neutrinos is based on the selection of cascade events generated by neutrino interactions in the sensitive volume of array. After applying an iterative procedure of vertex reconstruction followed by the rejection of hits contradicting the cascade hypothesis on each iteration stage, events with a final multiplicity of hit $\mathrm{OMs} \mathrm{N}_{\text {hit }}>20$ are selected as highenergy neutrino events. With this event selection, the neutrino effective areas for each flavor assuming an equal flux of neutrinos and antineutrinos and averaged over all arrival angles have been derived (see, Fig. 5, left). These areas are about ten times smaller than the corresponding areas of IceCube [11]. The accuracy of shower direction reconstruction is about 4 degree (median value), which is substantially better than the 10-15 degree accuracy for IceCube [11]. The fraction of shower events $\left(\mathrm{E}_{\mathrm{sh}}=100 \mathrm{TeV}\right)$ with mismatch angles between generated and reconstructed muon directions less than a given value is shown in Fig. 5 (right). Energy distributions of the expected number of shower events per year for IceCube astrophysical fluxes for different flavors and all-flavor, as well as the distribution of expected background events from atmospheric neutrinos are shown in Fig. 6 (left). The expected number of background 
events from atmospheric neutrinos is strongly suppressed for energies higher than $100 \mathrm{TeV}$. We expect about one event per year with $\mathrm{E}_{\mathrm{sh}}>100 \mathrm{TeV}$ from an all-flavor astrophysical flux with the normalization $\mathrm{E}^{2} \mathrm{~F}=3.6 \cdot 10^{-8} \mathrm{GeV} \mathrm{cm}^{-2} \mathrm{~s}^{-1} \mathrm{sr}^{-1}$ in the cluster, compared to about 10 events in IceCube. The sensitivity of cluster for one flavor neutrino flux with an $\mathrm{E}^{-2}$ spectrum and flavor ration 1:1:1 for all flavor flux as function of the observation years is shown in Fig. 6 (right). Three year exposition allows sensitivity at a level of flux value measured by IceCube.
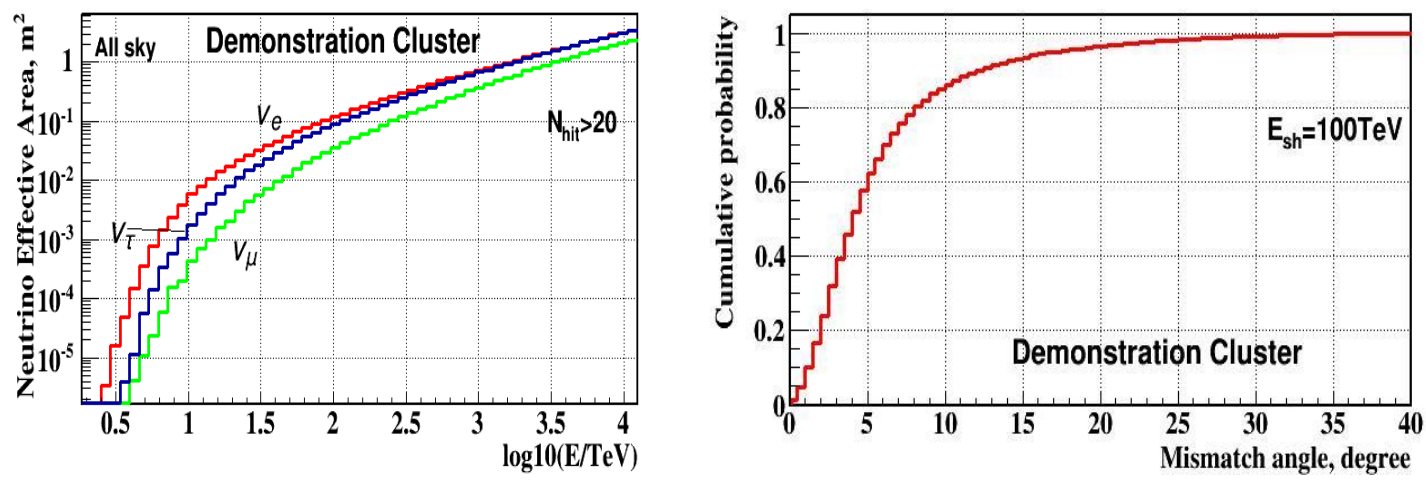

Fig. 5. Left: Neutrino effective areas of cluster for each flavor averaged over all arrival angles. The effective area includes effects from attenuation of neutrinos in Earth. Right: The fraction of reconstructed showers with mismatch angle less than a given value. Right: The fraction of reconstructed showers with mismatch angle less than a given value.
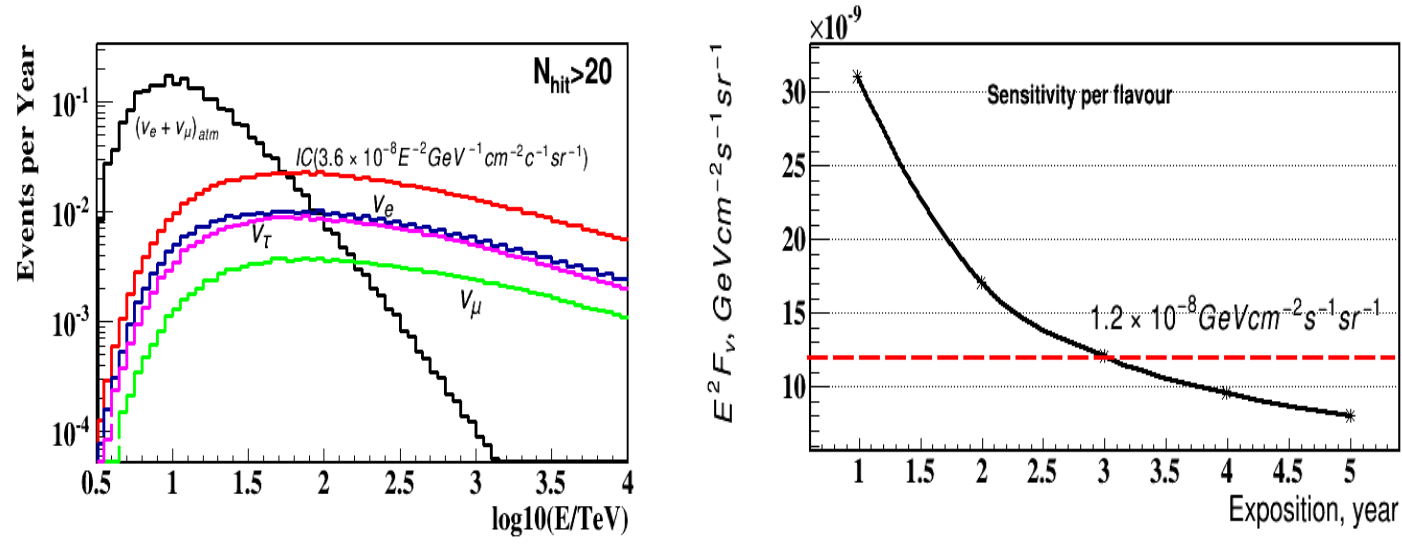

Fig. 6. Left: Expected distributions of events per year from astrophysical fluxes obtained by IceCube. Also shown is the expected distribution of background events from atmospheric neutrinos. Right: Cluster sensitivity for one flavor neutrino flux with an $\mathrm{E}^{-2}$ spectrum as function of the observation years. The long-dashed line indicates the one flavor neutrino flux value obtained by IceCube.

\section{Conclusion}

The ambition of the Baikal collaboration is the construction of a $\mathrm{km}^{3}$-scale neutrino telescope - the Gigaton Volume Detector in Lake Baikal. During the R\&D phase of the GVD project in 2008-2010, its basic elements - new optical modules, FADC readout units, underwater communications and trigger systems - have been developed, produced and tested in situ by long-term operating prototype strings. The prototyping phase of the GVD project has been performed since April 2011 and aims at comprehensive in situ test of all elements and 
systems of future detector. This phase is concluded with deployment and putting in operation of the first cluster of GVD in April 2015. For the years 2016-2020, the deployment of 10-12 clusters with about 2000 OMs is envisaged.

This work was supported by the Russian Foundation for Basic Research (grants 13-0212221, 14-02-00175, 14-02-00972).

\section{References}

[1] A. Avrorin et al., The prototyping/early construction phase of the BAIKAL-GVD project, NIM A742 82.

[2] A. Avrorin et al., Asp-15 - A stationary device for the measurement of the optical water properties at the NT200 neutrino telescope site, Nucl. Instr. Meth. A693 186-194.

[3] V. Aynutdinov et al., The prototype string for the km3-scale Baikal neutrino telescope, NIM $\mathbf{A 6 0 2}$ 227.

[4] V. Aynutdinov et al., The BAIKAL neutrino experiment, NIM A626 13.

[5] V. Aynutdinov et al., The gigaton volume detector in Lake Baikal, NIM A639 30.

[6] A. Avrorin et al., Current status of the Baikal-GVD project, NIM A725 23.

[7] A. Avrorin et al., Status and recent results of the Baikal-GVD project, Phys. of Part. and Nucl. 46211.

[8] A. Avrorin et al., A hydroacoustic positioning system for the experimental cluster of the cubickilometer-scale neutrino telescope at Lake Baikal, Instr. Exp.Tech. 56, 449.

[9] A. Avrorin et al., The optical module of the Baikal-GVD neutrino telescope, this Proceedings.

[10] A. Avrorin et al., Data acquisitions system of the NT1000 Baikal neutrino telescope, Instr. and Exp. Tech. 57262.

[11] M. G. Aartsen et al., Evidence for high-energy extraterrestrial neutrinos at the IceCube detector, Science 3421242856.

[12] M. G. Aartsen et al., Observation of high-energy astrophysical neutrinos in three years if IceCube data, Phys. Rev. Lett. 113101101. 